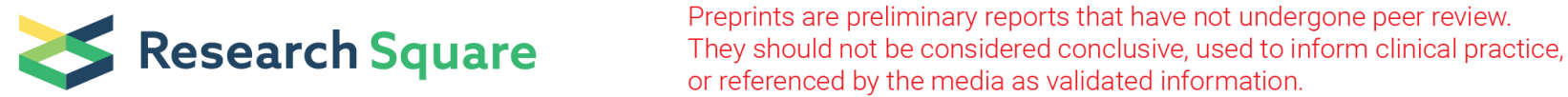

\section{Predicting the dropout risk after treatment of obesity: Logistic regression analysis and deep neural network analysis}

Tomohide Yamada ( $\sim$ bqx07367@yahoo.co.jp )

University of Tokyo

Chia-lin Lee

Taichung Veterans General Hospital

Wei-Ju Liu

Taichung Veterans General Hospital

Yuta Hiraike

Tokyo Daigaku

Jun Hosoe

University of Tokyo

Hisashi Noma

The institute of statistical mathematics

Nobuhiro Shojima

University of tokyo

\section{Methodology}

Keywords: obesity, comprehensive weight-loss programs, drop-out rates, machine learning

Posted Date: December 15th, 2019

DOI: https://doi.org/10.21203/rs.2.18781/v1

License: (c) (i) This work is licensed under a Creative Commons Attribution 4.0 International License.

Read Full License 


\section{Abstract}

Backgrounds

Severely obese patients must follow strict regimens of diet, exercise, and medical therapy. However, such comprehensive weight-loss programs have high dropout rates. In this study, we developed a machine learning prediction model to aid in the early detection of high-risk-dropout patients.

Methods

102 severely obese patients were monitored for 3 years to assess their risk of dropout from a comprehensive weight-loss program. The program targeted a $5 \%$ weight loss. It consisted of three main components, which include behavioral modification (goal setting and charting weight four times daily), diet, and exercise. A machine learning model was developed to predict dropout risk based on a 1-year dropout event. To extend the prediction ability past 1 year, we plotted a 3-year Kaplan-Meier survival curve using a deep learning (DL) algorithm and logistic regression (LR) classifications.

Results

Their mean age was $49 \pm 14$ years, $43 \%$ were male, BMI was $42 \mathrm{~kg} / \mathrm{m} 2$, and hemoglobin A1c was $7.6 \%$. Additionally, $76 \%$ had diabetes, $21 \%$ had impaired glucose tolerance. After 1 year, the dropout rate was $19 \%$. Using oral hypoglycemic agents had a lower risk of 3-year dropout [Odds ratio 0.26 ( $95 \% \mathrm{Cl}: 0.08$ to $0.83, p=0.023)$ ]. The area under the curve (AUC) was better with DL than LR methods for predicting dropout at 3 years $(0.97$ vs. $0.77, p<0.001)$. The AUC for DL was also better than LR using binary classifications ( 0.86 vs. $0.68, p=0.001)$.

Conclusions

We demonstrated a higher precision with machine learning than with the standard logistic regression, based on limited sample size and information available during hospital admission. It is vital to note that machine learning was more accurate than standard analysis. This may have clinical significance because machine learning could be used to identify high-risk groups and allow for early intervention.

\section{Introduction}

Obesity has rapidly become a worldwide epidemic and has nearly tripled since 1975 (1). In 2016, more than 1.9 billion adults, 18 years and older, were overweight. Of these over 650 million were obese. (1) Obesity and type 2 diabetes both increase the risk of cardiovascular disease and other comorbidities (2). A J-shaped relationship between body mass index (BMI) and the average life expectancy was demonstrated (3). Therefore, it is important to treat severe obesity, which significantly increases the risk of mortality. A slight weight loss (3\%-5\% of body weight) can result in clinically meaningful benefits of reducing triglycerides, blood glucose, glycated hemoglobin, and decreasing the risk of type 2 diabetes. An increased weight loss $(>5 \%)$ reduces blood pressure, further improves the lipid profile (both low-density 
and high-density lipoprotein cholesterol), and decreases the need for medications to control blood pressure, blood glucose, and lipids (4). Although many obese people successfully lose weight by dieting or behavioral therapy (using their preferred methods or one that is popular at the time), most of them subsequently regain the weight that they lost $(5,6)$. Long-term adherence to treatment is essential for obese patients; however, it is not easy to predict the discontinuation of treatment. Therefore, it is vital to control the progress of the severe complications caused by treatment interruption.

In this study, we aimed at developing an accurate prediction model on the risk of subsequent dropout from treatment by patients with severe obesity. All these patients had already completed a comprehensive weight-loss education program without bariatric surgery. We also developed a machine learning model to predict dropout and compared it with standard logistic regression by ROC analysis.

\section{Methods}

\section{Subjects}

First, we screened 2,178 successive patients who were admitted to the University of Tokyo Hospital between 2009 and 2012. Among them, we selected patients with severe obesity who had a BMI of more than $35 \mathrm{~kg} / \mathrm{m}^{2}$ and enrolled them in a comprehensive weight loss program $(5,6)$. We excluded patients with severe cardiovascular disease, heart failure, infectious diseases, stroke, or peripheral artery disease, as well as patients with type 1 diabetes, pregnant women, patients with dementia, patients who had orthopedic diseases that could interfere with exercise (walking), perioperative patients, patients taking anti-obesity medications, patients who had undergone bariatric surgery, patients without pertinent data, patients who were transferred to another hospital immediately after discharge, patients who were readmitted, and those under 18 or over 80 years old. The remaining patients were observed for 3 years after discharge to assess subsequent weight gain and dropout. The comprehensive inpatient obesity treatment program targeted a $5 \%$ weight loss; Its details have been reported previously (7). It consisted of three main components, which include behavioral modification (goal setting and charting weight four times daily), diet, and exercise (patients with diabetes received appropriate anti-diabetic therapy together with this weight loss program, and the treatment of diabetes was decided by each attending physician).

\section{Behavioral modification (goal setting and charting weight four times daily)}

At admission, all patients were given the goal of achieving $5 \%$ weight loss while in the hospital. They also weighed themselves four times daily (immediately after waking, taking breakfast and dinner, and before going to bed) and recorded the data on a weekly graph. A daily record of weight fluctuations reveals irregular intake of food and fluid that reflects dysfunctional eating patterns and other behavioral abnormalities and assists in achieving weight loss $(5,8,9)$. Patients were recommended to continue 
recording their weight after discharge. Patients were weighed by using AD-6107NWTM scales (A and D Co. Ltd., Tokyo, Japan).

\section{Diet}

A balanced low-calorie diet (20-24 kcal/day/kg of ideal body weight) was provided to the patients in the hospital, consisting of $50 \%-60 \%$ carbohydrate, $20 \%$ protein, and $20 \%-30 \%$ fat. Hospital dietitians used food samples and a food exchange table (10) to educate patients about nutritional guidelines. The dietician initially gave each patient information for 1 hour, with subsequent 30-minutes sessions being held at least twice a week until discharge.

\section{Exercise}

All patients were given a pedometer and were instructed to walk more than 10,000 steps/daily $(5-7 \mathrm{~km})$ for approximately 1.5 hours. The exercise program was tailored to accommodate health problems (e.g., morbid obesity, hypoglycemia, joint pain, or diabetic retinopathy) and specific needs (e.g., exercise by walking or training on a bicycle ergometer). The target pulse rate and schedule for each exercise session were set.

\section{Outcome measures}

The patients attended our hospital outpatient department every 2 months after discharge to continue their weight loss program and for treatment of other diseases (diabetes, dyslipidemia, and hypertension).

The body weight of the patients was measured at each visit. The objective of this study was to assess the dropout from the weight loss program after discharge. Dropout from the program was defined as missing outpatient appointments. (If the patient presented again within 6 months of the specified appointment, this was not considered as dropout.)

Patients were defined as having diabetes if their medical records listed a diagnosis of type 2 diabetes, and they were using an oral hypoglycemic agent or insulin. If a $75 \mathrm{~g}$ oral glucose tolerance test was performed, a diagnosis of diabetes, impaired glucose tolerance, or impaired fasting glucose was made according to the American Diabetes Association criteria (11). Antidepressant medications were classified as selective serotonin reuptake inhibitors, serotonin and norepinephrine reuptake inhibitors, tricyclic antidepressants, tetracyclic antidepressants, serotonin receptor antagonists and reuptake inhibitors, monoamine oxidase inhibitors, and noradrenergic and specific serotonergic antidepressants. All demographic and clinical data were collected from secure electric medical records. Nurses or physicians confirmed the accuracy of the bodyweight measurements of each subject 


\section{Feature engineering}

We used binary variables and continuous variables as prediction features. The binary variables included gender, use of oral antidiabetic drugs, use of insulin, use of Glucagon-like peptide-1 Receptor analogs (GLP1-Ras), diabetes, hypertension, psychiatric disease, depression, insomnia, and antipsychotic drug use. The continuous variable included sequential body weight data from baseline to day 14 , discharge body weight, age, waist circumference, systolic blood pressure, and HbA1C.

\section{Data preparation}

Overall, 102 patients (82 non-dropout and 20 dropout) were enrolled in this study. Due to the original imbalance sample between the dropout and non-dropout population, we used the Adaptive Synthetic Sampling (ADASYN) method to deal with the imbalanced data (12). In the balanced sample generated by the ADASYN method, there were 79 dropout and 82 non-dropout events. We randomized $85 \%$ of this balanced sample to a training cohort (for model training) and the rest of $15 \%$ as a validation cohort (for hyperparameter optimization). Then, we applied the deep learning (DL) algorithm generated from the balanced sample back to the original imbalanced cohort for individual prediction. The logistic regression (LR) model was also derived from the balanced sample and applied back to the original 102 people as individual risk prediction.

\section{Model building process of deep learning}

A machine learning model to predict the dropout rate was developed with deep neural networks. The variables derived from feature engineering (Table 1) were used as predictors and were set as input layers. For better performance of deep learning, we performed batch normalization (to mean 0 or variance 1) for the selected variables (features) and set this as the input layer. 
Table 1

Baseline characteristics between dropout and non-dropout at the end of the first year.

\begin{tabular}{|lllll|}
\hline & & & & \\
\hline Variable & Overall & Non-dropout(1y) & dropout(1y) & p-value \\
\hline N & 102 & 82 & 20 & \\
\hline Age (years old) & $49.3 \pm 14.1$ & $50.3 \pm 13.8$ & $45 \pm 15.1$ & 0.13 \\
\hline Male (\%) & $43(42)$ & $35(43)$ & $8(40)$ & 0.83 \\
\hline BMI & $42 \pm 9$ & $41 \pm 9$ & $41 \pm 5$ & 0.9 \\
\hline Wait circumstance (cm) & $123 \pm 13.2$ & $122 \pm 13.1$ & $126.9 \pm 12.9$ & 0.13 \\
\hline Systolic blood pressure (mmHg) & $131.3 \pm 17$ & $131.8 \pm 16.5$ & $129.2 \pm 19.4$ & 0.54 \\
\hline HbA1c (\%) & $7.6 \pm 2.5$ & $7.7 \pm 2.4$ & $6.8 \pm 2.5$ & 0.13 \\
\hline Type 2 diabetes (\%) & $78(76.47)$ & $67(81.71)$ & $11(55)$ & 0.01 \\
\hline Hypertension (\%) & $68(66.67)$ & $59(71.95)$ & $10(50)$ & 0.06 \\
\hline Psychosis (\%) & $41(40.2)$ & $33(40.24)$ & $8(40)$ & 0.98 \\
\hline Depression (\%) & $14(13.73)$ & $11(13.41)$ & $3(15)$ & 0.85 \\
\hline Insomnia (\%) & $24(23.53)$ & $21(25.61)$ & $3(15)$ & 0.32 \\
\hline Other mental disease (\%) & $25(24.51)$ & $19(23.17)$ & $6(30)$ & 0.52 \\
\hline Oral hypoglycemic agent (\%) & $51(50)$ & $45(54.88)$ & $6(30)$ & 0.046 \\
\hline Insulin therapy (\%) & $23(22.55)$ & $20(24.39)$ & $3(15)$ & 0.37 \\
\hline Sulfonylurea & $5(4.9)$ & $5(6.1)$ & $0(0)$ & 0.26 \\
\hline GLP-1 analogs & $14(13.73)$ & $13(15.85)$ & $1(5)$ & 0.21 \\
\hline
\end{tabular}

The entire structure of the deep neural network was designed as follows: 25 input layers $\rightarrow 25$ middle hidden layers $\rightarrow 25$ middle hidden layers $\rightarrow 20$ middle hidden layers $\rightarrow 16$ middle hidden layers $\rightarrow$ onedimensional output layer. The binary outcome of the 1-year dropout or non-dropout was set as the output layer. To avoid overfitting during the model training of deep learning, we used an L1 regularization method (13), as well as adding a dropout layer between the hidden layers. The dropout rate was set at 0.2. As activation functions, we employed scaled exponential linear units in the middle layer and hard sigmoid units in the output layer.

\section{Statistics}


The independent $t$ test and $\chi 2$ test were used to compare continuous and categorical variables between dropout and non-dropout subgroups. To compare the model performance between $D L$ and LR, we calculated the area under the receiver operating characteristic curve (ROC) based on the 1-year dropout event in the original cohort $(\mathrm{N}=102)$. To see the extension of the prediction ability after 1 year, we plotted the 3-year survival curve using the Kaplan-Meir method according to DL and LR classification. We used a TensorFlow version 1.10.0. for model building. Logistic regression analysis was performed with Scikitlearn version 0.20.3. Machine learning was done with Python version 3.6.5 (Python Software Foundation, Hampton, NH). Statistical analyses were performed using the SAS software (Version 9.4; SAS Institute, Inc., Cary, NC, USA).

\section{Results}

Figure 1 is a flow chart showing the disposition of the patients. A total of 2,178 consecutive adults with severe obesity were admitted to our hospital between January 2009 and December 2012. Among them, 2,010 were excluded because their BMI was less than $35 \mathrm{~kg} / \mathrm{m}^{2}$. Among the remaining 168 patients, 66 were excluded ( 21 because of readmission to our department to treat diabetes and obesity, 11 because of emergency admission, 11 because of transfer to another hospital immediately after discharge, and 23 because of lack of pertinent data). The remaining 102 patients were included in this study.

Table 1 shows the baseline characteristics between dropout and non-dropout at the end of the first year.

Their mean age was $49 \pm 14$ years, $43 \%$ were male, BMI was $42 \mathrm{~kg} / \mathrm{m}^{2}$, and hemoglobin A1c was $7.6 \%$. Additionally, $76 \%$ had diabetes, $21 \%$ had impaired glucose tolerance. Over a follow-up period of 1 year after discharge, 20 of the 102 patients (19\%) dropped out of the program. The dropout subgroup showed a much lower proportion of diabetic patients and less anti-diabetic medication than the non-dropout subgroup. Multivariate LR analysis showed that using oral hypoglycemic agents had a significantly lower risk of 3-year dropout [odds ratio 0.26 ( $95 \% \mathrm{Cl} 0.08$ to $0.83, \mathrm{p}=0.023$ )]. In addition, waist circumstance was significantly associated [odds ratio 1.06 ( $95 \% \mathrm{Cl}: 1.01$ to $1.1, \mathrm{p}=0.019)$ ].

Figure 2A shows the area under the receiver operating characteristic curve (ROC) between LR and DL in the prediction of the 1-year dropout. DL showed a much better AUC than the LR method ( 0.97 vs. $0.77, p$ for difference $<0.001$ ). During clinical practice, physicians tried to identify more people at high risk of dropout. Therefore, we tried to identify an optimal threshold to keep the sensitivity $>80 \%$ and yield the highest value of specificity. We used this concept of threshold selection rather than the traditional methods, such as the Yuden index (14). Finally, a predicted probability greater than 0.4 was used as the threshold to define a high risk of dropout after one year. The AUC using binary classification between LR and DL was plotted in Fig. 2B. The DL showed better AUC than the LR (0.86 vs. 0.68 . $p$ for difference $=$ $0.001)$.

Figure $3 \mathrm{~A}$ shows the overall survival curve between the true dropout and non-dropout subgroups. Figure 3B showed the 3-year survival curve of the subgroups classified by LR predicted the 1-year dropout 
and non-dropout. Similarly, Fig. 3C showed the predicted survival curve categorized by DL. Both LR and DL methods showed significant differences between the predicted dropout and non-dropout after the first year and continued estrangement for 3 years.

Generally, the dropout rate was $0.9 / 100$ patients per month. However, the dropout rate was $14.3,4.1$, and 1.9 /100 patients per month for subgroups with the true-dropout, DL, and LR predicted dropout. The subgroup of DL predicted dropout showed a significantly higher dropout incidence rate than the subgroup predicted by LR ( $p=0.03$, data not shown). Meanwhile, the corresponding dropout rate was $0,0.2$, and $0.3 / 100$ patients per month for subgroups of true non-dropout, $D L$, and LR predicted non-dropout. In comparison with the true non-dropout subgroup, the LR and DL predicted non-dropout did not show higher dropout incidence rates ( $p=0.982$ and 0.983 , respectively).

\section{Discussion}

This study demonstrates the superiority of our neural network model over logistic regression for predicting the dropout from a comprehensive program of severe obesity after discharge from the hospital.

There is notable clinical significance from these analyses. Firstly, dropout is a critical issue; however, it is challenging to predict it using a single parameter, especially in a small sample size study. Secondly, the deep Learning model showed higher AUC than logistic regression. The deep learning model showed a higher dropout prediction than logistic regression; however, it still had a lower prediction as compared with the true dropout $(p=0.01)$. Deep learning and logistic regression showed almost similar predicted non-dropout rates, compared with the true non-dropout rate.

There were some limitations to this study. Firstly, DL tends to model overfitting. We had used two methods (dropout and L1 Regularization) to reduce overfitting; however, external validation will be required in future studies. Secondly, the small sample size used will require external validation.

\section{Conclusions}

We demonstrated a higher precision with machine learning than with the standard logistic regression, based on limited sample size and information available during hospital admission. It is vital to note that machine learning was more accurate than standard analysis. This may have clinical significance because machine learning could be used to identify high-risk groups and allow for early intervention.

\section{List Of Abbreviations}

DL deep learning

LR logistic regression 
AUC area under the curve

BMI body mass index

GLP1-Ras glucagon-like peptide-1 receptor analogs

\section{Declarations}

\section{Ethics approval and consent to participate}

This retrospective cohort study complied with the Declaration of Helsinki and it was approved by the Research Ethics Committee of the University of Tokyo Hospital.

\section{Consent for publication}

Not applicable

\section{Availability of data and materials}

The datasets used and/or analysed during the current study are available from the corresponding author on reasonable request.

\section{Competing interests}

The authors declare that they have no competing interests.

\section{Funding}

There was no funding in this study.

\section{Author contributions}

T.Y., L.C., L.W. extracted and interpreted the data. T.Y., and L.C., drafted the manuscript. T.Y., L.C., L.W., Y.H., J.H., H.N., and N.S. contributed the discussion and approved submission of this manuscript for publication.

\section{Acknowledgements}

Not applicable

\section{References}

1. World Health Organization. WHO Obesity and overweight 2018. [WWW document]. Available from https://www.who.int/news-room/fact-sheets/detail/obesity-and-overweight; 2018. Accessed 4 Dec 2019. 
2. Mozaffarian D. Dietary and Policy Priorities for Cardiovascular Disease, Diabetes, and Obesity: A Comprehensive Review. Circulation. 2016 Jan 12;133(2):187-225.

3. Bhaskaran K, Dos-Santos-Silva I, Leon DA, Douglas IJ, Smeeth L. Association of BMI with overall and cause-specific mortality: a population-based cohort study of 3.6 million adults in the UK. Lancet Diabetes Endocrinol. 2018 Dec;6(12):944-953.

4. Jensen MD, Ryan DH, Apovian CM, Ard JD, Comuzzie AG, Donato KA, Hu FB, Hubbard VS, Jakicic JM, Kushner RF, Loria CM, Millen BE, Nonas CA, Pi-Sunyer FX, Stevens J, Stevens VJ, Wadden TA, Wolfe BM, Yanovski SZ; American College of Cardiology/American Heart Association Task Force on Practice Guidelines; Obesity Society. 2013 AHA/ACC/TOS guideline for the management of overweight and obesity in adults: a report of the American College of Cardiology/American Heart Association Task Force on Practice Guidelines and The Obesity Society. J Am Coll Cardiol. 2014 Jul 1;63(25 Pt B):2985-3023

5. Yamada T, Hara K, Svensson AK, Shojima N, Hosoe J, Iwasaki M, Yamauchi T, Kadowaki T. Successfully achieving target weight loss influences subsequent maintenance of lower weight and dropout from treatment. Obesity (Silver Spring). 2015 Jan;23(1):183-91

6. Yamada T, Lee CL, Liu WJ, Shojima N. Slow Weight Loss During Comprehensive Treatment and Worse Metabolic Control with Higher Weight Regain: A Trajectory Analysis. Obesity (Silver Spring). 2019 Dec;27(12):1925-1926.

7. lizuka Y, Ohashi K, Tsukamoto K, Ueki K, Kadowaki T. Education and management of diabetes at The University of Tokyo Hospital. J Diabetes 2011;3(2): 104-108.

8. Yamada T, Kiuchi Y, Nemoto M, Yamashita S. Charting weight four times daily as an effective behavioural approach to obesity in patients with type 2 diabetes. Diab Vasc Dis Res 2014;11(2):118120.

9. Fujimoto $\mathrm{K}$, Sakata $\mathrm{T}$, Etou $\mathrm{H}$, et al. Charting of daily weight pattern reinforces maintenance of weight reduction in moderately obese patients. Am J Med Sci 1992; 303(3):145-150.

10. The Japan Diabetes Society. Food Exchange Lists-Dietary Guidance for Persons with Diabetes. Bunkyo-do: The Japan Diabetes Society; 2002.

11. American Diabetes Association. 2. Classification and Diagnosis of Diabetes: Standards of Medical Care in Diabetes-2018. Diabetes Care. 2018 Jan;41(Suppl 1):S13-S27.

12. He H, Bai Y, Garcia EA, Li S. ADASYN: Adaptive synthetic sampling approach for imbalanced learning. IEEE International Joint Conference on Neural Networks, Hong Kong, 2008, 1322-1328.

13. Yuan GX, Chang KW, Hsieh CJ, Lin CJ. A Comparison of Optimization Methods and Software for Large-scale L1-regularized Linear Classification. Journal of Machine Learning Research 2010;11:3183-3234.

14. Ruopp MD, Perkins NJ, Whitcomb BW, Schisterman EF. Youden Index and optimal cut-point estimated from observations affected by a lower limit of detection. Biom J. 2008 Jun;50(3):419-30.

\section{Figures}




\section{8 patients admitted to our hospital from 2009 to 2012}

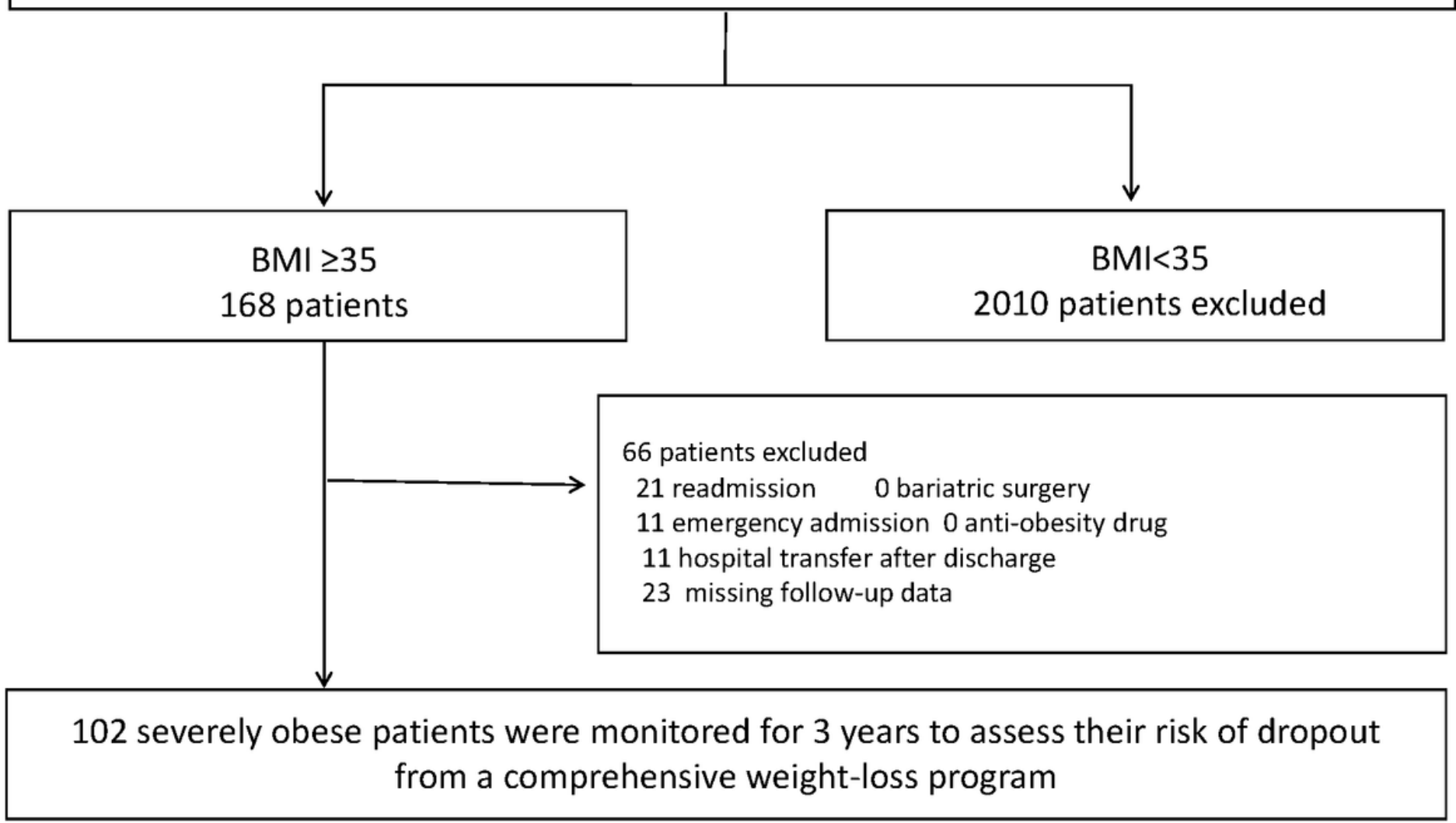

\section{Figure 1}

Flow chart of patient disposition. 


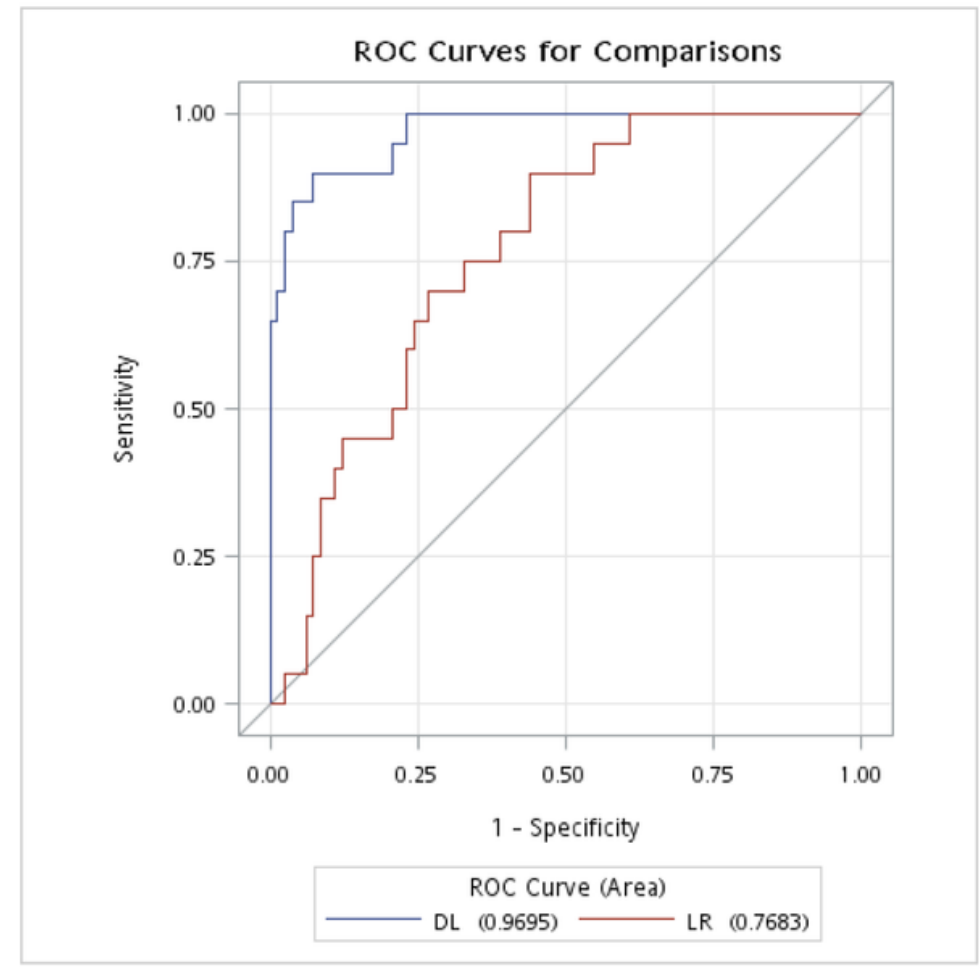

Figure $2 \mathrm{~A}$

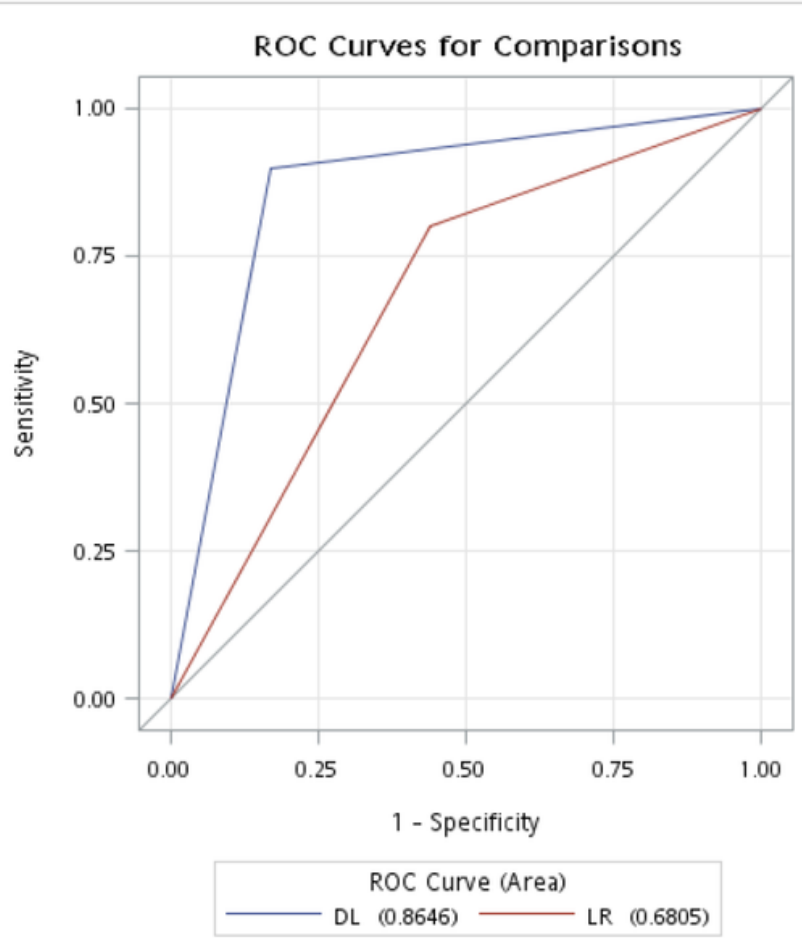

Figure 2B

\section{Figure 2}

Area under the receiver operating characteristic curve (ROC) between LR and DL in the prediction of the 1year dropout.
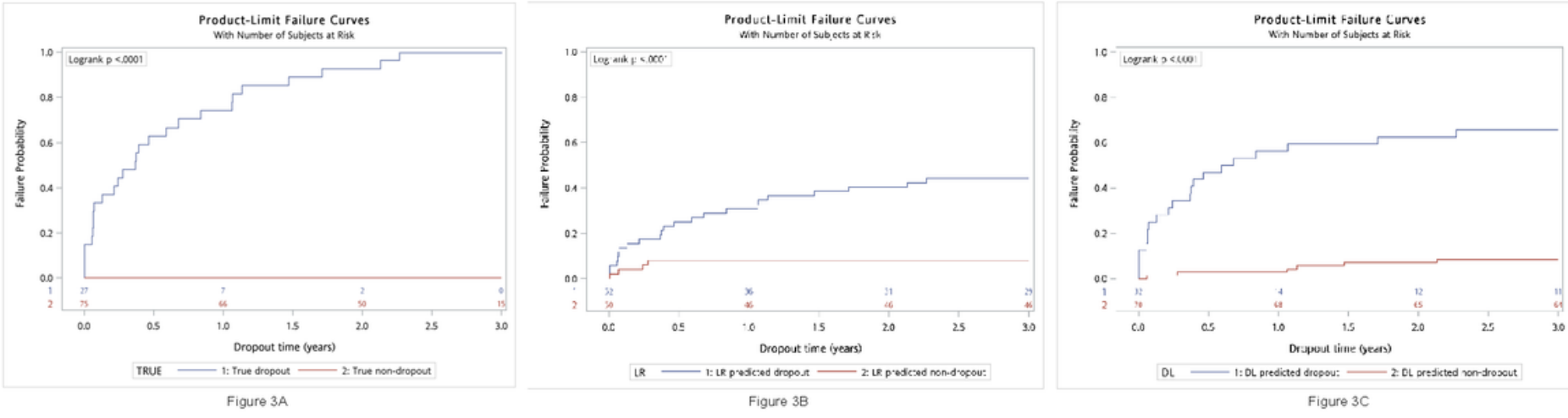

\section{Figure 3}

Survival curves of true dropout and non-dropout subgroups.

\section{Supplementary Files}

This is a list of supplementary files associated with this preprint. Click to download.

- STROBEchecklistcohort.pdf 